\title{
Adsorption Enhanced Lubrication by Thin Liquid Films of Synthetic and Biological Polyelectrolyte Solutions
}

\author{
Akira TAKAHASHI and Norio KOZAKI \\ Department of Industrial Chemistry, Faculty of Engineering, \\ Mie University, Tsu, Mie 514, Japan
}

(Received November 25, 1986)

\begin{abstract}
Lubrication properties of three polyelectrolytes such as $\mathrm{Na}$-poly(acrylate), $\mathrm{Na}-$ chondroitin sulphate, and $\mathrm{Na}$-hyaluronate were studied by measuring the static friction coefficient $\mu_{\mathrm{s}}$ of thin liquid films of these aqueous polyelectrolyte solutions between glass surfaces. Adsorption isotherms of the polyelectrolytes onto the glass surface were also measured. Thin liquid films of polyelectrolyte solutions with added $\mathrm{NaCl}$ definitely show lubricity. The $\mu_{\mathrm{s}} v s$. bulk concentration curves have relevance to the adsorption isotherm and lead to the conclusion that the adsorbed polyelectrolyte layer has the ability to reduce the $\mu_{\mathrm{s}}$ value of glass-water-glass interface to the extent of $40-60 \%$. Among the polyelectrolytes tested, $\mathrm{Na}$-hyaluronate has the lowest $\mu_{\mathrm{s}}$ owing to that this polyelectrolyte is used in the animal joints as the lubricant.

KEY WORDS Adsorption of Polyelectrolyte / Lubrication / Polyelectrolyte / Poly(acrylic) Acid / Chondroitin Sulphate / Hyaluronic Acid / Tribology / Static Friction Coefficient /
\end{abstract}

The lubrication action of biological polyelectrolyte, particularly hyaluronic acid in animal joints is well known. Studies have been reported to intend exploring molecular mechanism of biological lubrication in the joints. ${ }^{1-3}$ However, as far as we are aware, no reports have been published concerning with the lubrication action of thin liquid films of both synthetic and biological polyelectrolyte solutions. In the previous papers, ${ }^{4,5}$ we reported the lubrication properties of water soluble nonionic polymers by measuring the static friction coefficient $\mu_{\mathrm{s}}$ of thin liquid films of the polymer solutions between glass surfaces. This paper is the extension of the previous investigation to explore lubricating mechanism of polyelectrolyte. To clarify the correlation between lubrication properties of polyelectrolyte solutions and the polyelectrolyte adsorption on the glass surface, both $\mu_{\mathrm{s}}$ and the adsorption isotherms are measured using $\mathrm{Na}$-poly(acrylate), $\mathrm{Na}$-chondroitin sulphate, and $\mathrm{Na}-$ hyaluronate, and the results are discussed from the view point of boundary lubrication assisted by the adsorbed polyelectrolyte layer.

\section{EXPERIMENTAL}

\section{Samples}

Fractionated sodium poly(acrylate) (NaPA) was used in this study. Its molecular weight was determined from intrinsic viscosity measurement according to the equation ${ }^{6}$

$$
\begin{gathered}
{[\eta]=12.4 \times 10^{-4} \bar{M}_{w}{ }^{0.5}} \\
\left(1.5 \mathrm{M} \mathrm{NaBr}, 15^{\circ} \mathrm{C}\right)
\end{gathered}
$$

The characteristic of $\mathrm{Na}-\mathrm{PA}$ is shown in Table I.

Sodium chondroitin sulphate $(\mathrm{Na}-\mathrm{Ch})$ wäs purchased from Wako Pure Chemicals and sodium hyaluronate ( $\mathrm{Na}-\mathrm{Hyal})$ was supplied from Tokyo Kasei Co. The characteristic of 
Table I. Characteristics of polyelectrolytes

\begin{tabular}{lc}
\hline Polyelectrolyte & $\bar{M}_{w} \times 10^{-3}$ \\
\hline Na-Poly(acrylate) & 597 \\
Na-Chondroitin sulphate & - \\
Na-Hyaluronate & $700^{\mathrm{a}}$ \\
\hline
\end{tabular}

a Supplier's data.

$\mathrm{Na}-\mathrm{Hyal}$ is also shown in Table I. These biological polyelectrolytes were used without further purification, and to avoid degradation, sodium azide $\left(\mathrm{NaN}_{3}\right)(0.02 \mathrm{wt} \%)$ was added in their aqueous solutions. Twice distilled water was used as solvent. Sodium chloride (Nakarai Chemical Co.) was used as an added salt.

\section{Static Friction Coefficient}

Static friction coefficient $\mu_{\mathrm{s}}$ of each polyelectrolyte solution in the presence or absence of $\mathrm{NaCl}$ were measured by the same instrument as that described previously. ${ }^{4}$ Watch glasses and optical flat plate glasses supplied from Central Glass Co., Japan were first dipped into a warm $\mathrm{H}_{2} \mathrm{SO}_{4}-\mathrm{HNO}_{3}$ (1: 1 vol. ratio) mixture, then thoroughly rinsed in distilled water and dried in a dust free box. For each polyelectrolyte concentration and load $W$ at least eleven measurement of frictional force $F$ were performed and then the average value of $F$ was used to determine $\mu_{\mathrm{s}}$ from eq 2

$$
\mu_{\mathrm{s}}=F / W
$$

\section{Absorption of Polyelectrolyte on Glass Sur- face}

Spherical and pore free glass beads with a diameter $\phi=17 \mu \mathrm{m}$ and specific surface area $=1.41 \times 10^{3} \mathrm{~cm}^{-2} \mathrm{~g}^{-1}$ supplied from Toshiba Ballotini Co., Japan were used as the adsorbent. Beads were washed by benzene in a Soxhlet apparatus for $6 \mathrm{~h}$, dryed, immersed in aqueous $0.1 \mathrm{~N} \mathrm{HCl}$ solution and thoroughly rinsed by distilled water. After drying the beads at $80^{\circ} \mathrm{C}$ for $24 \mathrm{~h}$, they were immersed in methanol, washed by distilled $n$-hexane and finally dried in a vacuum oven for $48 \mathrm{~h}$ at $130^{\circ} \mathrm{C}$.

Seven milliliters of an aqueous salt solution of polyelectrolyte with known concentration were mixed with the glass beads $(1 \mathrm{~g})$ in a stoppered polycarbonate tube. The mixture was gently stirred by a magnetic chip for $24 \mathrm{~h}$ to attain equilibrium at $25^{\circ} \mathrm{C}$. The glass suspensions were centrifuged at $5,000 \mathrm{rpm}$ for 20 min to sediment the glass beads and the supernatant was carefully removed. The polymer concentration in the supernatant, i.e., the equilibrium bulk concentration, $C_{\mathrm{p}}$ was determined as follows: $C_{\mathrm{p}}$ was measured from the peak area of each polyelectrolyte using a Toyo Soda HLC-802A gel permeation chromatography instrument equipped by a TSK RI 8 detector. The TSKG 6000 PW column was used.

Adsorbance A expressed in $\mathrm{g} \mathrm{cm}^{-2}$, was determined from the difference between the amount of the polyelectrolyte initially added $C_{\mathrm{o}}$ and that in the supernatant $C_{\mathrm{p}}$, and also from the amount of the glass beads added.

\section{RESULTS AND DISCUSSION}

As an example, Figure 1 shows the static friction coefficient $\mu_{\mathrm{s}}$ of the aqueous $\mathrm{Na}-\mathrm{PA}$ solution (concentration: $0.1 \mathrm{~g} / 100 \mathrm{ml}$ ) with added $\mathrm{NaCl}(0.1 \mathrm{~N})$ as a function of load $(W)$. The $\mu_{\mathrm{s}}$ value decreases with an increase in $W$ below $W=100 \mathrm{~g}$ and it becomes constant above $W=100 \mathrm{~g}$ in accordance with Coulomb's friction law. ${ }^{7}$ All following measurements of $\mu_{\mathrm{s}}$ were carried out at $W=$ $150 \mathrm{~g}$.

\section{$\mathrm{Na}-\mathrm{Poly}$ (acrylate) $-\mathrm{NaCl}-\mathrm{H}_{2} \mathrm{O}$ System}

In Figure 2 , the $\mu_{\mathrm{s}}$ values of $\mathrm{Na}-\mathrm{PA}$ solutions are plotted against polymer concentration $C_{\mathrm{p}}$, both in the presence and absence of $\mathrm{NaCl}$. The $\mu_{\mathrm{s}}$ values indicated by a square in the figure are constant $(0.50 \pm 0.01)$ independent of the $\mathrm{NaCl}$ concentration. The $\mu_{\mathrm{s}}$ of $\mathrm{Na}-$ 


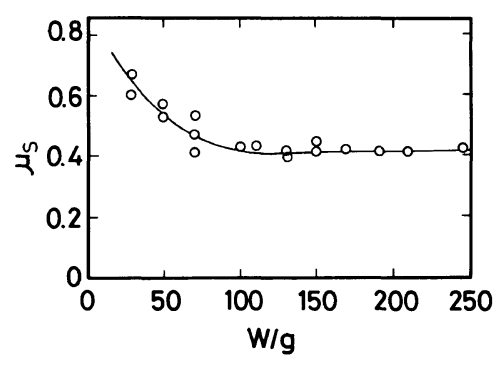

Figure 1. Static friction coefficient $v s$. load $W$ plots for a Na-PA solution. Polymer concentration, $0.1 \mathrm{~g} / 100 \mathrm{ml}$; $\mathrm{NaCl}$ concentration, $0.1 N$.

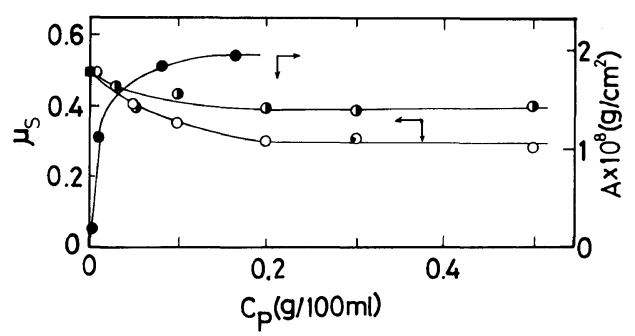

Figure 2. Polymer concentration dependence of $\mu_{\mathrm{s}}$ of Na-PA. $\left(\bar{M}_{w}=597 \times 10^{3}\right) . \square, \mathrm{H}_{2} \mathrm{O}$ and $0.1 \mathrm{~N} \mathrm{NaCl}$; $\mathrm{NaCl}$ concentration $\left(C_{\mathrm{s}}{ }^{\circ}\right) ; \bigcirc, C_{\mathrm{s}}{ }^{\circ}=0 ; \boldsymbol{D}, 0.1 N$ and plots of adsorption isotherm of $\mathrm{Na}-\mathrm{PA}$ on glass beads in $0.1 \mathrm{~N}$ $\mathrm{NaCl}$ at $25^{\circ} \mathrm{C}$.

PA solutions first decreased with increasing polymer concentration, then became independent of $C_{p}$. In the plateau region, the $\mu_{\mathrm{s}}$ decreased with increase of the salt concentration. Moreover, these $\mu_{\mathrm{s}}$ values were almost the same values for water soluble nonionic polymers as previously reported. ${ }^{4}$

The adsorption isotherm of $\mathrm{Na}-\mathrm{PA}$ onto the glass beads is also displayed in Figure 2. We can see a correlation between the adsorption isotherm and friction coefficient-concentration curve, that is, $\mu_{\mathrm{s}}$ is high at very small adsorbance $A$, then with increasing of $A, \mu_{\mathrm{s}}$ decreased, and at the plateau region of $A \mu_{\mathrm{s}}$ became constant.

$\mathrm{Na}-$ Chondroitin Sulphate- $\mathrm{NaCl}-\mathrm{H}_{2} \mathrm{O}$ System and $\mathrm{Na}$-Hyaluronate- $\mathrm{NaCl}-\mathrm{H}_{2} \mathrm{O}$ System

The repeating structure of chondroitin sulphate and hyaluronic acid are shown in Figure

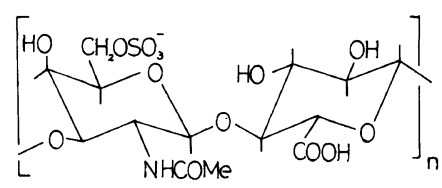

(a)

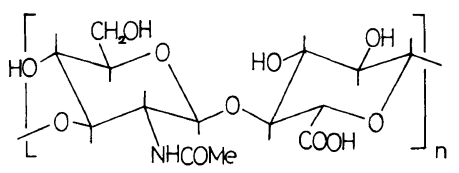

(b)

Figure 3. The repeating structure of: (a) chondroitin sulphate, and (b) hyaluronic acid.

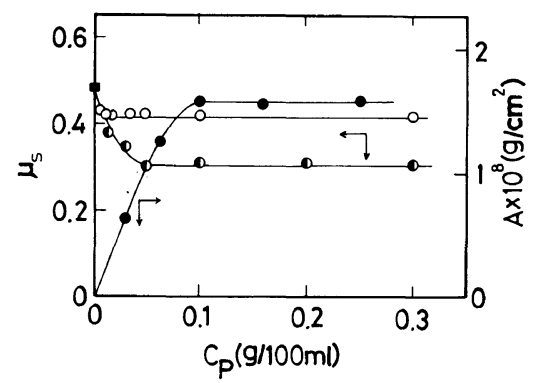

Figure 4. Polymer concentration dependence of $\mu_{\mathrm{s}}$ of $\mathrm{Na}$-chondroithin sulphate in water $(\mathrm{O})$ and physiological saline solution $(\mathrm{O})$ at $25^{\circ} \mathrm{C} . \square, \mathrm{H}_{2} \mathrm{O}$ and the saline solution, and plots of adsorption isotherm of $\mathrm{Na}-\mathrm{Ch}$ on the glass beads from the physiological saline solution at $25^{\circ} \mathrm{C}$.

3 , the former is a sulphate containing strong polyelectrolyte, while the latter is a carboxy containing weak polyelectrolyte.

The concentration dependence of $\mu_{\mathrm{s}}$ values and the adsorption isotherm of $\mathrm{Na}-\mathrm{Ch}$ are shown in Figure 4. It is clear that this animal origin polyelectrolyte also exhibits static friction coefficient reduction and we observe also the correlation between the adsorption isotherm and the friction coefficient-concentration curve. Comparison between salt free and physiological saline solutions shows that the addition of $\mathrm{NaCl}$ reduces $\mu_{\mathrm{s}}$ further, however, $\mu_{\mathrm{s}}$ values do not differ from those of $\mathrm{Na}-\mathrm{PA}$ in salt solution.

$\mathrm{Na}-\mathrm{Hyal}$, which is an another animal origin 


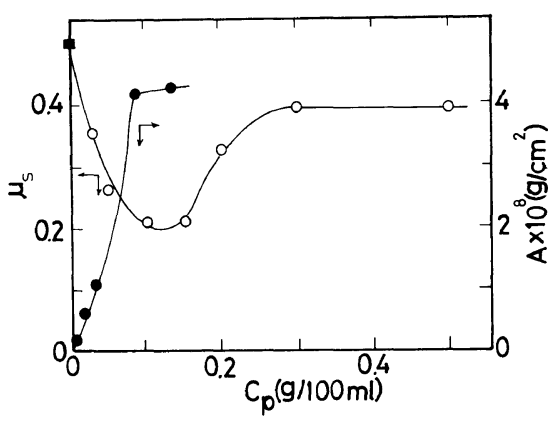

Figure 5. Polymer concentration dependence of $\mu_{\mathrm{s}}$ of $\mathrm{Na}$-hyaluronate in physiological saline solution at $25^{\circ} \mathrm{C}$.

$\square$, saline solution, and plots of adsorption isotherm of $\mathrm{Na}-\mathrm{Hyal}$ on the glass beads from physiological saline solution at $25^{\circ} \mathrm{C}$.

polysaccharide is a very interesting polyelectrolyte since it is not only a typical glycosaminoglycan but also a unique friction reduction polymer in animal joints. The concentration dependence of $\mu_{\mathrm{s}}$ and the adsorption isotherm of $\mathrm{Na}-\mathrm{Hyal}$ are shown in Figure 5. The $\mu_{\mathrm{s}}$ values first decreased with increasing $C_{\mathrm{p}}$, increased after showing a minimum $\mu_{\mathrm{s}}$, and then leveled off. The minimum $\mu_{\mathrm{s}}$ value is the lowest among the examined polyelectrolytes and clearly shows the unique property of $\mathrm{Na}-$ Hyal as mentioned above. Above $C_{\mathrm{p}}=0.2 \mathrm{~g} /$ $100 \mathrm{ml}, \mathrm{Na}-\mathrm{Hyal}-$ aqueous $\mathrm{NaCl}$ solution becomes very viscous and exhibits gelation, therefore, $\mu_{\mathrm{s}}$ values become high. Until the minimum $\mu_{\mathrm{s}}$ value which occurs around $C_{\mathrm{p}}=$ $0.1-0.15 \mathrm{~g} / 100 \mathrm{ml}$, adsorption isotherm could be measured, however, at the higher bulk concentration than $C_{\mathrm{p}}=0.15 \mathrm{~g} / 100 \mathrm{ml}$ the adsorbance could not be measured because of considerable gelation or association of $\mathrm{Na}-\mathrm{Hyal}$ which probably caused by hydrogen bonds as infered from its repeating structure. Above $C_{\mathrm{p}}=0.3 \mathrm{~g} / 100 \mathrm{ml}$ the friction mode would be changed from the boundary lubrication to fluid or viscous lubrication.

It is clear from the above results the existence of closer correlation between the static friction coefficient of thin liquid films of the polyelectrolytes solution-concentration relationship and the adsorption isotherms of the polyelectrolytes.

Bowden and Tabor $^{8}$ proposed a contact model for the mechanism of boundary lubrication caused by adsorbed molecular film. According to them, since the true contact area between solids $S$ is usually not covered completely by the adsorbed film, static friction coefficient $\mu_{\mathrm{s}}$ is given by introducing the fraction of uncovered area, $\alpha$

$$
\begin{aligned}
\mu_{\mathrm{s}} & =F / W=(1 / W)\left\{S \alpha \sigma_{\mathrm{M}}+S(1-\alpha) \sigma_{\mathrm{L}}\right\} \\
& =1 / p\left[\alpha \sigma_{\mathrm{M}}+(1-\alpha) \sigma_{\mathrm{L}}\right]
\end{aligned}
$$

where $p=W / S, \sigma_{\mathrm{M}}$ and $\sigma_{\mathrm{L}}$ are the shearing strengths of solid, and adsorbed film, respectively. Therefore to reduce $\mu_{\mathrm{s}}$, the necessary conditions are as follows: (a) $\sigma_{\mathrm{L}}<\sigma_{\mathrm{M}}$ and (b) $\alpha$ should be as small as possible. In general, $\sigma_{\mathrm{L}}<\sigma_{\mathrm{M}}$ since adsorbed molecular film is much softer than the solid. The adsorption isotherms of tested polyelectrolytes onto glass surfaces are the high affinity type, which is common for polymer and polyelectrolyte adsorption on solid surfaces. ${ }^{9}$ In the very low bulk polymer concentration region, adsorbance and the fraction of occupied sites $\theta$ which may be correlated with $(1-\alpha)$ are small and the adsorbed polymer chains take a flat conformation consist of alternative small loops and trains. Thus the high $\alpha$ value results. In general, with increasing the bulk polymer concentration adsorbance as well as $\theta$ increase and simultaneously, the adsorbed layer thickness also increases, thus the fraction of uncovered area $\alpha$ will be decreased. Further increase of polymer concentration induces that both the adsorbance and $\theta$ become constant and adsorbed layer thickness also becomes constant, thus resulting constant $\alpha$. Therefore, the steep increase of adsorbance means the steep reduction of uncovered surface fraction $\alpha$ and at the plateau region of the adsorption isotherm $\alpha$ is very small but reaches a constant value. Hence, $\mu_{\mathrm{s}}$ first steeply decreases and then be- 
comes constant, and the coincidence of transition region of $\mu_{\mathrm{s}} v s . C_{\mathrm{p}}$ plots and adsorption isotherm also supports this explanation.

As we have already shown, ${ }^{10-12}$ the adsorbance of polyelectrolyte on the uncharged surface and/or the slightly charged surface, which has the same sign of charge of the polyelectrolyte, decreases with the decrease of salt concentration, since electrostatic repulsive force among adsorbed polyions prevents further adsorption. Thus, the adsorbance of polyelectrolyte in salt free system is much smaller than that of salt added system and results higher $\mu_{\mathrm{s}}$ values than that in salt added system as shown in Figures 2 and 4.

It is of much interest that the existence of the minimum $\mu_{\mathrm{s}}$ in the friction coefficients of $\mathrm{Na}$ -Hyal vs. $C_{\mathrm{p}}$ curve and that the fact $\mathrm{Na}-\mathrm{Hyal}$ is selected as the lubricant of animal joints. However, at the present stage we cannot explain why the unique structure of $\mathrm{Na}-\mathrm{Hyal}$ is most effective in reducing of $\mu_{\mathrm{s}}$.

In conclusion, thin liquid films of aqueous polyelectrolyte solutions definitely have a lubricating function and the adsorption of polyelectrolyte on solid surfaces is responsible for this function. $\mathrm{Na}-\mathrm{Hyaluronate}$, which is the unique and only lubricant employed by the animal joint has the lowest friction coefficient at a specified bulk concentration.

\section{REFERENCES}

1. P. R. Lewis and C. W. McCutchen, Nature, 184, 1285 (1959).

2. V. Wright, D. Dowson, and J. Kerr, Int. Rev. Connective Tissue Res., 6, 105 (1973).

3. A. D. Roberts, J. Colloid Interface Sci., 41, 23 (1972).

4. T. Kato, N. Kozaki, and A. Takahashi, Polym. J., 18, 111 (1986).

5. T. Kato, N. Kozaki, and A. Takahashi, Polym. J., 18, 189 (1986).

6. A. Takahashi and M. Nagasawa, J. Am. Chem. Soc., 86, 543 (1964).

7. C. A. Coulomb, "Memoeires de Mathématiquie et de Physique de l'Academie Royale des Sciences," Paris, 1785, p 161.

8. F. P. Bowden and D. Tabor, "The Friction and Lubrication of Solid," Part II. The Clarendon Press, Oxford, 1950; "The Friction and Lubrication of Solid," Oxford University Press, Boston, 1964.

9. A. Takahashi and M. Kawaguchi, Adv. Polym. Sci., 46, 1 (1982).

10. A. Takahashi, M. Kawaguchi, and T. Kato, Polymer Science and Technology, Vol. 12, "Adhesion and Adsorption of Polymers," Part B, L-H, Lee Ed., Plenum Press, New York, N.Y., 1980, p 729.

11. A. Takahashi, M. Kawaguchi, K. Hayashi, and T. Kato, "Polymer Adsorption and Dispersion Stability," E. D. Goddard and B. Vincent Ed., Am. Chem. Soc. Symp. Ser. No. 240, 1984, p 39.

12. M. Kawaguchi, K. Hayashi, and A. Takahashi, Macromolecules, 17, 2066 (1984). 